\title{
Variações baseadas em gênero no movimento e na força isométrica do tronco em jovens adultos com dor lombar: Um estudo de caso-controle prospectivo
}

\section{Gender-based Variations in Trunk Motion and Isometric Strength in Young Adults with Low Back Pain: A Prospective Case-control Study}

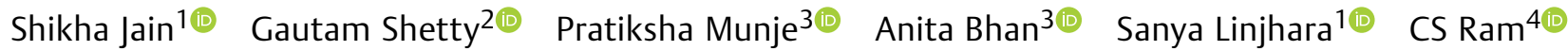

1 Departmento de Fisioterapia, QI Spine Clinic, Deli, Índia

${ }^{2}$ Departmento de Cirurgia Ortopédica, Knee \& Orthopaedic Clinic,

Mumbai; Diretor de Pesquisa, QI Spine Clinic, Mumbai, Índia

Endereço para correspondência Dr. Gautam Shetty, MBBS, MSOrth, QI India Healthcare, \#6 Level 2 Phoenix Market City, LBS Road, Kamani,

${ }^{3}$ Departmento de Fisioterapia da Coluna, QI Spine Clinic, Deli, Índia Kurla (West), Mumbai 400070, Índia

${ }^{4}$ Departamento de Fisioterapia, I.T.S Faculdade de Fisioterapia, Ghaziabad, Uttar Pradesh, Índia

Rev Bras Ortop 2022;57(3):392-401.

\begin{abstract}
Resumo
\section{Palavras-chave}

- dor lombar

- força muscular

- amplitude de movimento articular

- contração isométrica

- coluna vertebral

Objetivo Determinar as variações na amplitude de movimento (ADM) do tronco e na força isométrica do tronco (FIT) em jovens adultos sintomáticos e assintomáticos baseadas no gênero dos indivíduos.

Métodos Neste estudo caso-controle prospectivo, 73 indivíduos com dor lombar (DL) e 80 indivíduos assintomáticos foram analisados. As medidas de ADM do tronco e FIT de extensão, flexão e rotação foram comparadas em ambos os grupos e em subgrupos organizados por gênero. A análise multivariada foi usada para determinar os fatores que influenciam a ADM do tronco e a FIT.

Results Symptomatic males had significantly less extension RoM and extension, flexion, and rotation isometric trunk strength (ITS) $(p<0.0001)$ compared with asymptomatic males, whereas no significant difference was found between asymptomatic and symptomatic females. However, the mean extension-flexion RoM and mean extension-flexion ITS ratios were significantly lower $(p=0.04)$ in asymptomatic females compared with symptomatic females. Female gender was significantly associated with less extension and flexion ITS in both asymptomatic and symptomatic subjects.

Conclusão Indivíduos do sexo masculino com DL apresentaram significativa fraqueza global relacionada à FIT quando comparados com indivíduos do sexo masculino assintomáticos. Apesar de não haver diferença significativa de FIT em indivíduos do
\end{abstract}

Estudo conduzido na QI Spine Clinic, Índia.

recebido

04 de Março de 2021

aceito

02 de Junho de 2021

Publicado on-line

Setembro 11, 2021
DOI https://doi.org/

10.1055/s-0041-1736199.

ISSN 0102-3616.
(C) 2021. Sociedade Brasileira de Ortopedia e Traumatologia. All rights reserved.

This is an open access article published by Thieme under the terms of the Creative Commons Attribution-NonDerivative-NonCommercial-License, permitting copying and reproduction so long as the original work is given appropriate credit. Contents may not be used for commercial purposes, or adapted, remixed, transformed or built upon. (https://creativecommons.org/ licenses/by-nc-nd/4.0/)

Thieme Revinter Publicações Ltda., Rua do Matoso 170, Rio de Janeiro, RJ, CEP 20270-135, Brazil 


\begin{abstract}
Keywords

- low back pain

- muscle strength

- range of motion, articular

- isometric contraction

- spine

Objective To determine gender-based variations in trunk range of motion (RoM) and isometric strength (IS) in symptomatic and asymptomatic young adults.

Methods In this prospective case-control study, 73 subjects with low back pain (LBP) and 80 asymptomatic subjects were analyzed. Dynamometer-based device trunk RoM and IS measurements in extension, flexion, and rotation were compared in both groups and gender-based subgroups. Multivariate analysis was used to determine factors influencing trunk RoM and IS.

Results Symptomatic males had significantly less extension RoM and extension, flexion, and rotation isometric trunk strength (ITS) $(p<0.0001)$ compared with asymptomatic males, whereas no significant difference was found between asymptomatic and symptomatic females. However, the mean extension-flexion RoM and mean extension-flexion ITS ratios in females were significantly lower $(p=0.04)$ in asymptomatic compared with symptomatic females. Female gender was significantly associated with less extension and flexion ITS in both asymptomatic and symptomatic subjects.

Conclusion Males with LBP had significant global ITS weakness when compared with asymptomatic males. Despite no significant ITS difference in symptomatic versus asymptomatic females, LBP caused significant extension-flexion RoM and ITS imbalance in females. These gender-based variations in trunk RoM and IS, especially the extensor-flexor IS imbalance in females, must be considered while designing rehabilitation treatment protocols for LBP.
\end{abstract}

sexo feminino sintomáticos versus assintomáticos, a DL impactou a ADM e a FIT de extensão-flexão em indivíduos do sexo feminino. Essas variações de ADM do tronco e FIT baseadas no sexo, especialmente o desequilíbrio extensor-flexor de força isométrica em indivíduos do sexo feminino, devem ser consideradas ao projetar-se protocolos de tratamento de reabilitação para lombalgia.

\section{Introdução}

A disfunção e a fraqueza dos músculos do tronco são fatores de risco importantes para o desenvolvimento da dor lombar (DL) ${ }^{1-3} \mathrm{~A}$ força do músculo do tronco compensa a disfunção causada pela DL, e a redução da amplitude de movimento (ADM) do tronco e da força muscular pode levar a limitações funcionais e incapacidade em indivíduos com DL. ${ }^{2,4}$ Portanto, restaurar a força muscular e a ADM do tronco utilizando exercícios ajuda a reduzir a dor e a incapacidade, assim como a melhorar a função e prevenir a recorrência em pacientes com DL. ${ }^{5}$ Além disso, a melhora da ADM da lombar ou do tronco e da força lombar é um parâmetro útil para medir a eficácia do tratamento de reabilitação em pacientes com DL. ${ }^{6,7}$ Portanto, medir a ADM do tronco e a força isométrica do tronco (FIT) em um paciente com DL ajudará a quantificar as debilidades na ADM e força do tronco, individualizar o tratamento de reabilitação com base nessas debilidades e registrar a melhora com o tratamento.

A força muscular dos músculos paravertebrais e a mobilidade do tronco podem variar com base nas características do indivíduo, como sexo, idade, duração dos sintomas, intensidade da dor e nível de debilidade. ${ }^{1,8-10}$ Estudos anteriores sobre a ADM do tronco e da FIT avaliaram especificamente a força do músculo extensor lombar ou do tronco, em vez da ADM global do tronco e da força isométrica, e mediram esses parâmetros em uma ampla faixa de idade. $^{1,8-10}$

Até onde sabemos, nenhum estudo na literatura investigou especificamente a diferença na ADM do tronco e na FIT entre indivíduos sintomáticos e assintomáticos no grupo na idade vulnerável de 20 a 40 anos, e investigou a diferença baseada no gênero na ADM do tronco e FIT entre sintomáticos com DL e indivíduos assintomáticos nesta faixa etária. Acreditamos que a determinação de variações específicas relacionadas ao gênero nos parâmetros de ADM do tronco e FIT ajudará a projetar protocolos de tratamento de reabilitação específicos para pacientes com base nessas debilidades em pacientes submetidos ao tratamento conservador da DL. Portanto, este estudo teve como objetivo comparar a ADM do tronco e a FIT em indivíduos com DL e indivíduos assintomáticos semelhantes, comparar a ADM do tronco e a FIT em indivíduos sintomáticos e assintomáticos dos sexos masculino e feminino e determinar os fatores que influenciam a ADM do tronco e a FIT nos grupos sintomáticos e assintomáticos. Nossa hipótese era que haveria uma diferença 
significativa entre indivíduos sintomáticos e assintomáticos, tanto em indivíduos do sexo masculino quanto feminino, em termos de ADM de tronco e FIT.

\section{Métodos}

\section{Desenho do estudo}

Este estudo comparativo prospectivo foi conduzido em uma rede de clínicas especializadas em reabilitação da coluna vertebral, de abril de 2019 a março de 2020. Os participantes foram divididos entre grupo de DL sintomática (caso) e grupo assintomático (controle), e então comparados neste estudo. $\mathrm{O}$ protocolo do estudo foi aprovado por um conselho de revisão institucional e pelo comitê de ética, e todos os participantes assinaram um termo de consentimento para participação neste estudo.

\section{Participantes}

Os indivíduos no grupo sintomático (DL) foram recrutados de grupos de pacientes que vieram para avaliação de sua DL e foram submetidos a teste de ADM e FIT em um de nossas três clínicas de reabilitação de coluna em uma cidade. Um total de 149 indivíduos com DL que foram consecutivamente submetidos a teste de ADM do tronco e FIT durante o período de estudo foram elegíveis para participação no grupo sintomático. Indivíduos assintomáticos foram recrutados entre os parentes dos pacientes do grupo sintomático e entre os fisioterapeutas que trabalham em qualquer uma das nossas 8 clínicas de reabilitação da coluna em três cidades. Um total de 84 voluntários assintomáticos que consentiram com o teste de ADM do tronco e FIT foram elegíveis para participação no estudo como parte do grupo assintomático.

O critério de inclusão para o grupo sintomático foi pacientes que se apresentaram na clínica para avaliação de lombalgia mecânica. Os critérios de exclusão foram pacientes $<20$ anos ou $>40$ anos de idade, envolvimento de articulações periféricas, deformidades cifóticas ou escolióticas estruturais, cirurgia prévia da coluna vertebral e prontuários clínicos incompletos. O critério de inclusão para o grupo assintomático foi participantes sem DL ou quaisquer sintomas musculoesqueléticos dentro do último ano. Os critérios de exclusão para o grupo assintomático foram pacientes $<20$ anos ou $>40$ anos de idade, histórico de trauma na coluna, tuberculose óssea na coluna e deformidades cifóticas/escolióticas.

\section{Avaliação clínica}

Depois de registrado o histórico clínico, todos os indivíduos foram examinados quanto à postura, ADM lombar, teste de elevação da perna estendida (SLR, na sigla em inglês) e avaliação dos dermátomos e dos miótomos. Com base no histórico clínico e nos exames, os indivíduos com DL que apresentavam dor na coluna, nos discos intervertebrais ou nos tecidos moles circundantes, dor que piorava com movimento específico da coluna e melhorava com repouso, foram diagnosticados com lombalgia mecânica. ${ }^{11}$ No grupo sintomático, a dor foi medida usando a pontuação da escala de avaliação numérica da dor (NPRS, na sigla em inglês). ${ }^{12}$

\section{Protocolo de teste de ADM do tronco e FIT}

A ADM do tronco e a FIT foram testadas em todos os indivíduos usando um dinamômetro segundo protocolo descrito previamente. ${ }^{13}$ Segundo relatos anteriores, a confiabilidade e a reprodutibilidade do teste de força do tronco utilizando este equipamento foram classificadas de boas a excelentes. ${ }^{13}$ Todos os participantes foram testados em três dispositivos separados (para extensão, flexão e rotação) na posição sentada e presos no lugar usando um sistema de bloqueio de joelhos e um cinto de restrição de coxa que imobilizava o quadril e a coxa, e permitia o movimento apenas do tronco e da parte inferior das costas. Para iniciar o teste, o tronco foi travado em $30^{\circ}$ de flexão a partir da posição sentada ereta para extensão, colocado em posição neutra para flexão e a parte inferior do corpo rotacionado $30^{\circ}$ no plano transversal, confirmado visualmente na tela do aparelho. Após o aquecimento inicial, todos os participantes geraram sua contração isométrica máxima, aumentando gradualmente seu momento de força até o máximo nos primeiros 2 a 3 segundos de cada contração. 0 melhor valor obtido em 3 tentativas foi registrado. Os intervalos entre as repetições ou tentativas máximas de teste foram de no mínimo 15 segundos. Os 3 testes isométricos máximos diferentes (extensão, flexão e rotação) foram espaçados por 5 minutos. Toda a avaliação da força foi realizada sob a supervisão de um fisioterapeuta de coluna treinado e experiente na utilização do equipamento. A ADM do tronco foi medida em todos os três dispositivos sequencialmente após o término do teste específico de FIT. Os valores de força (torque) e movimento (graus) foram capturados pelo software do dispositivo e armazenados em seu servidor.

\section{Medidas dos resultados}

Dados demográficos, incluindo sexo, idade, índice de massa corporal (IMC), estilo de vida e duração dos sintomas (agudo/subagudo $<12$ semanas, crônico $\geq 12$ semanas) foram coletados em todos os participantes. As medidas de ADM do tronco, extensão máxima, flexão, rotação direita e rotação esquerda foram registradas em graus. Para a FIT, o torque máximo para extensão, flexão, rotação à direita e rotação à esquerda do tronco foi registrado em $\mathrm{Nm}$. A relação de extensão-flexão de ADM foi calculada dividindo o valor máximo de ADM de extensão (graus) pelo valor máximo de ADM de flexão (graus), e a proporção de extensão-flexão foi calculada dividindo o valor de força máxima de extensão $(\mathrm{Nm})$ pelo valor de força máxima de flexão $(\mathrm{Nm})$ para determinar o desequilíbrio de extensão-flexão de ADM e FIT.

\section{Análise estatística}

Com base nos achados de força muscular isométrica no movimento de flexão média em um teste piloto inicial com 10 indivíduos, para uma diferença de $20 \%$ na força muscular isométrica de flexão entre os 2 grupos, valor $\alpha$ de 0,05 e potência em $80 \%$, um tamanho de amostra mínimo de 63 indivíduos foi calculado em cada grupo usando a calculadora de tamanho de amostra ClinCalc (ClinCalc LLC, Indiana, EUA). Os dados categóricos foram comparados com o teste do qui- 
quadrado, e os dados contínuos foram comparados com a análise de variância (ANOVA, na sigla em inglês) de uma via entre os grupos sintomáticos e assintomáticos e os subgrupos com base no sexo. Uma análise multivariada foi realizada para determinar o efeito da idade, gênero, IMC, estilo de vida e duração dos sintomas nos movimentos de extensão e flexão da ADM e da FIT em indivíduos assintomáticos e sintomáticos. Um valor de $p<0,05$ foi considerado significativo. A análise estatística foi realizada usando a ferramenta de análise estatística online GraphPad QuickCalcs (GraphPad Software, San Diego, CA, EUA).

\section{Resultados}

Com base nos critérios de exclusão, 76 indivíduos foram excluídos do grupo de DL sintomática (12 indivíduos com registros clínicos incompletos e 64 indivíduos que estavam fora da faixa etária de 20 a 40 anos) e 4 indivíduos foram excluídos do grupo assintomático ou controle (1 indivíduo com histórico de tuberculose óssea e 3 indivíduos que estavam fora da faixa etária de 20 a 40 anos). Portanto, foram analisados dados de 73 indivíduos do grupo sintomático com DL (40 homens e 33 mulheres) e 80 indivíduos do grupo assintomático (24 homens e 56 mulheres). As características de todos os indivíduos estão resumidas na -Tabela 1.

\section{Comparação da ADM do tronco e da FIT em indivíduos do sexo masculino sintomáticos e assintomáticos}

Em indivíduos do sexo masculino, a ADM do tronco média em movimento de extensão foi significativamente menor $(p<0,0001)$ em indivíduos sintomáticos quando comparados com indivíduos assintomáticos ( - Tabela 2 e - Fig. 1), e a força média do tronco nos movimentos de extensão $(p<0.0001)$, flexão $(p<0.0001)$, e rotação $(p<0.0001)$ foi significativamente menor em indivíduos sintomáticos quando comparados com indivíduos assintomáticos (-Tabela 2 e -Fig. 2). No entanto, a média de ADMs de flexão e rotação, e a média da amplitude de extensão-flexão $(p=0,09)$ e a média da força de extensão-flexão $(p=0,55)$ não foram significativamente diferentes entre os 2 subgrupos (-Tabela 2 and -Fig. 1).

\section{Comparação de ADM do tronco e da FIT em indivíduos do sexo feminino sintomáticos e assintomáticos}

Em indivíduos do sexo feminino a ADM média de flexão do tronco foi significativamente menor em indivíduos sintomáticos quando comparados com indivíduos assintomáticos (-Tabela 2 e - Fig. 1). A média de ADM de extensão-flexão $(p=0,04)$ e a razão média de extensão-flexão $(p=0,04)$ foram significativamente maiores em indivíduos sintomáticos quando comparados com indivíduos assintomáticos. No entanto, não houve diferença significativa para a ADM média de extensão do tronco e ADM de rotação e para a força média de extensão, flexão e rotação do tronco entre os 2 subgrupos (-Tabela 2 e -Figs. 1, 2).

Fatores que afetam a ADM do tronco e a FIT em indivíduos assintomáticos e sintomáticos
Em indivíduos assintomáticos, a análise multivariada mostrou que sexo feminino e maior IMC foram significativamente associados com menor ADM de extensão do tronco, enquanto nenhum fator foi encontrado para afetar significativamente a ADM de flexão (- Tabela 3). Para a FIT, o sexo feminino e o menor IMC foram significativamente associados à menor força de extensão do tronco, enquanto o sexo feminino foi significativamente associado à menor força de flexão do tronco (-Tabela 3). Em indivíduos com DL, a análise multivariada mostrou que nenhum fator afetou significativamente a ADM de extensão e flexão, enquanto o sexo feminino e idade mais avançada foram significativamente associados com menor FIT de movimento de extensão, e o sexo feminino foi significativamente associado com menor FIT em movimento de flexão ( - Tabela 4 ).

\section{Discussão}

Os resultados deste estudo indicam que, embora os indivíduos do sexo masculino sintomáticos tivessem significativamente menor ADM de extensão de tronco média e FIT média em comparação com indivíduos do sexo masculino assintomáticos, não houve diferença significativa na ADM de extensão de tronco e FIT quando indivíduos do sexo feminino assintomáticos e sintomáticos foram comparados. No entanto, a média de amplitude de extensão-flexão e as razões médias de FIT de extensão-flexão no sexo feminino foram significativamente menores em indivíduos assintomáticos quando comparados com indivíduos do sexo feminino sintomáticos. $\mathrm{O}$ sexo feminino foi significativamente associado com menor FIT de extensão e flexão em indivíduos sintomáticos e assintomáticos.

Uma comparação anterior baseada no gênero entre indivíduos sintomáticos e assintomáticos não mostrou diferença na FIT em movimento de extensão em indivíduos do sexo masculino, ao passo que foi significativamente maior em indivíduos do sexo feminino assintomáticos quando comparados com indivíduos do sexo feminino com lombalgia crônica. ${ }^{8}$ Esses achados são contrários aos resultados do presente estudo. Isso poderia ser explicado por um maior número de indivíduos no grupo sintomático, uma faixa etária mais ampla da população do estudo (18-90 anos), inclusão apenas de lombalgia crônica e recrutamento de indivíduos assintomáticos da comunidade em geral em seu estudo. ${ }^{8}$

Em contraste com os indivíduos do sexo masculino, não foi encontrada diferença significativa na ADM de extensão do tronco e na FIT entre indivíduos do sexo feminino assintomáticos e sintomáticos no presente estudo. Da mesma forma, em contraste com os indivíduos do sexo masculino, a média da ADM de extensão-flexão e as relações médias de força de extensão-flexão em indivíduos do sexo feminino foram significativamente menores em indivíduos assintomáticos quando comparados com indivíduos sintomáticos. A razão de força de extensão-flexão indica um desequilíbrio antagonista/agonista aumentado, e uma razão mais alta indica FIT dos flexores menor em relação à FIT dos extensores. Portanto, esse achado indica que em indivíduos do sexo feminino com DL, a fraqueza da extensão individual do 


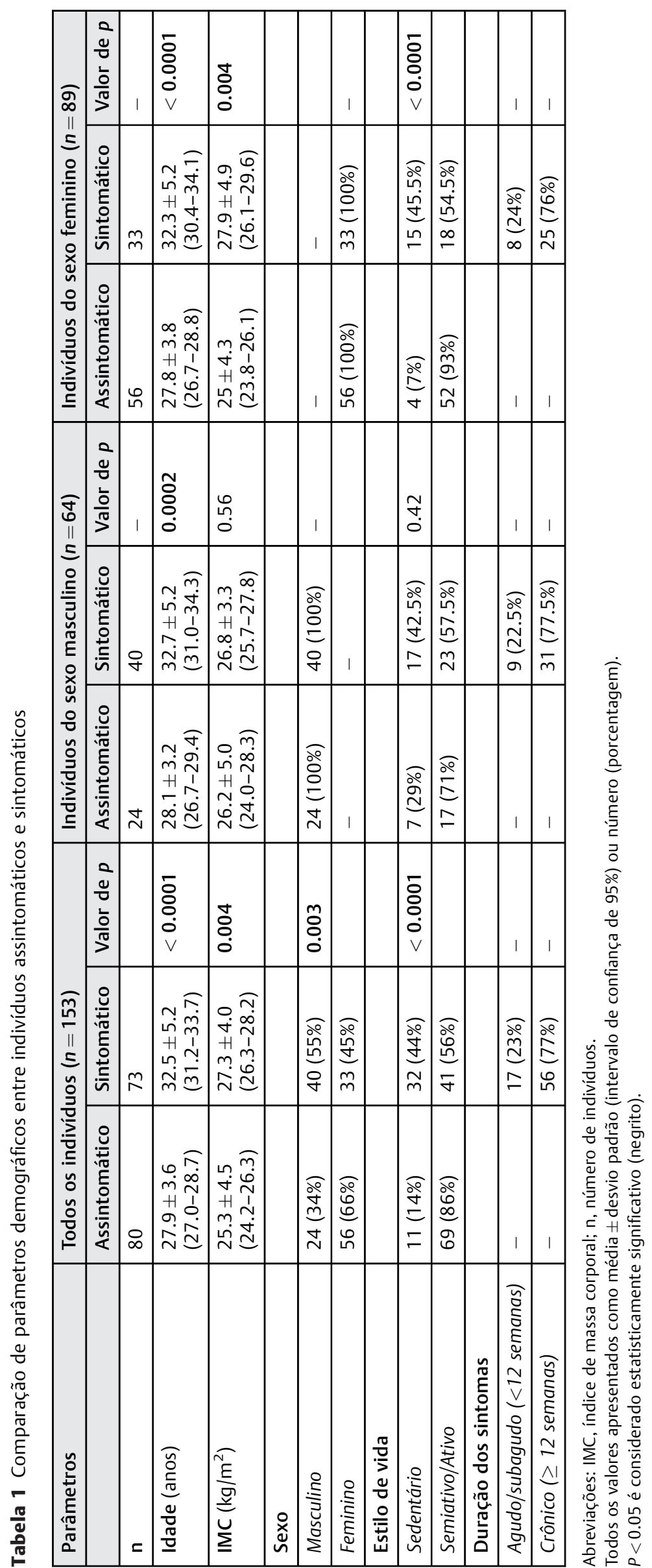




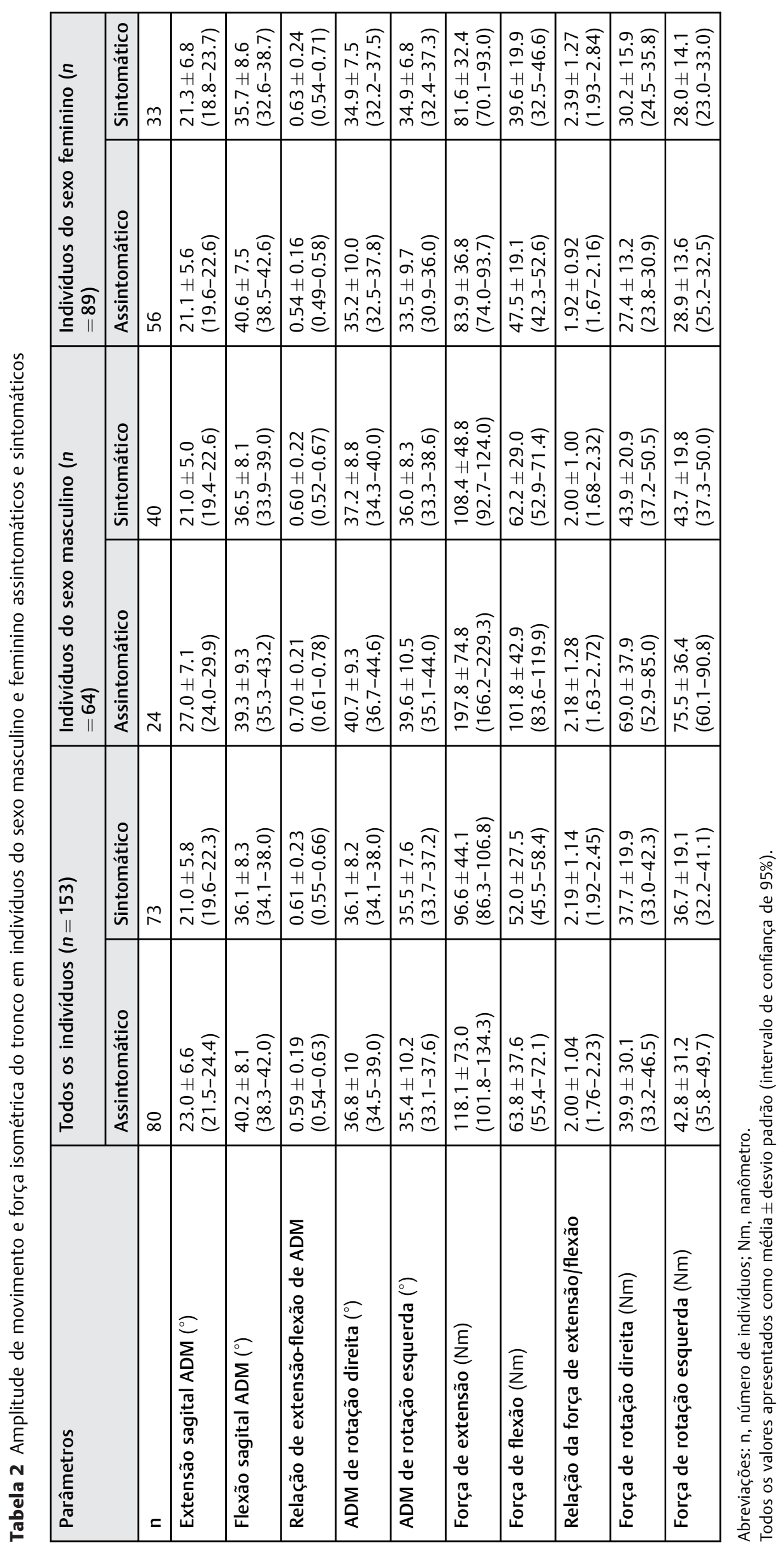


398 Variações baseadas em gênero em jovens adultos com dor lombar Jain et al.

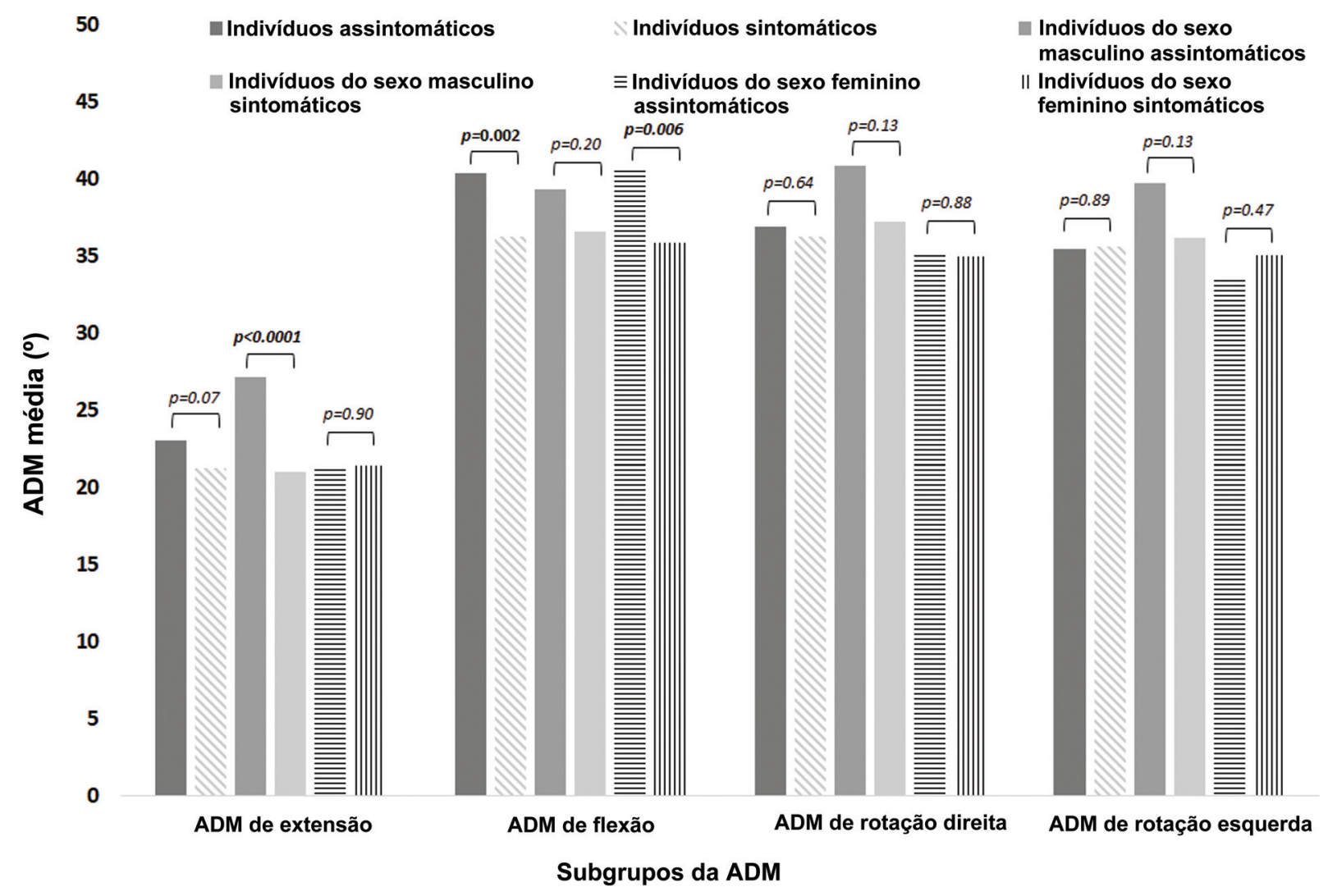

Fig. 1 Comparação da amplitude de movimento (ADM) do tronco média entre indivíduos do sexo masculino e feminino assintomáticos e sintomáticos. $P<0,05$ é considerado estatisticamente significativo (negrito).

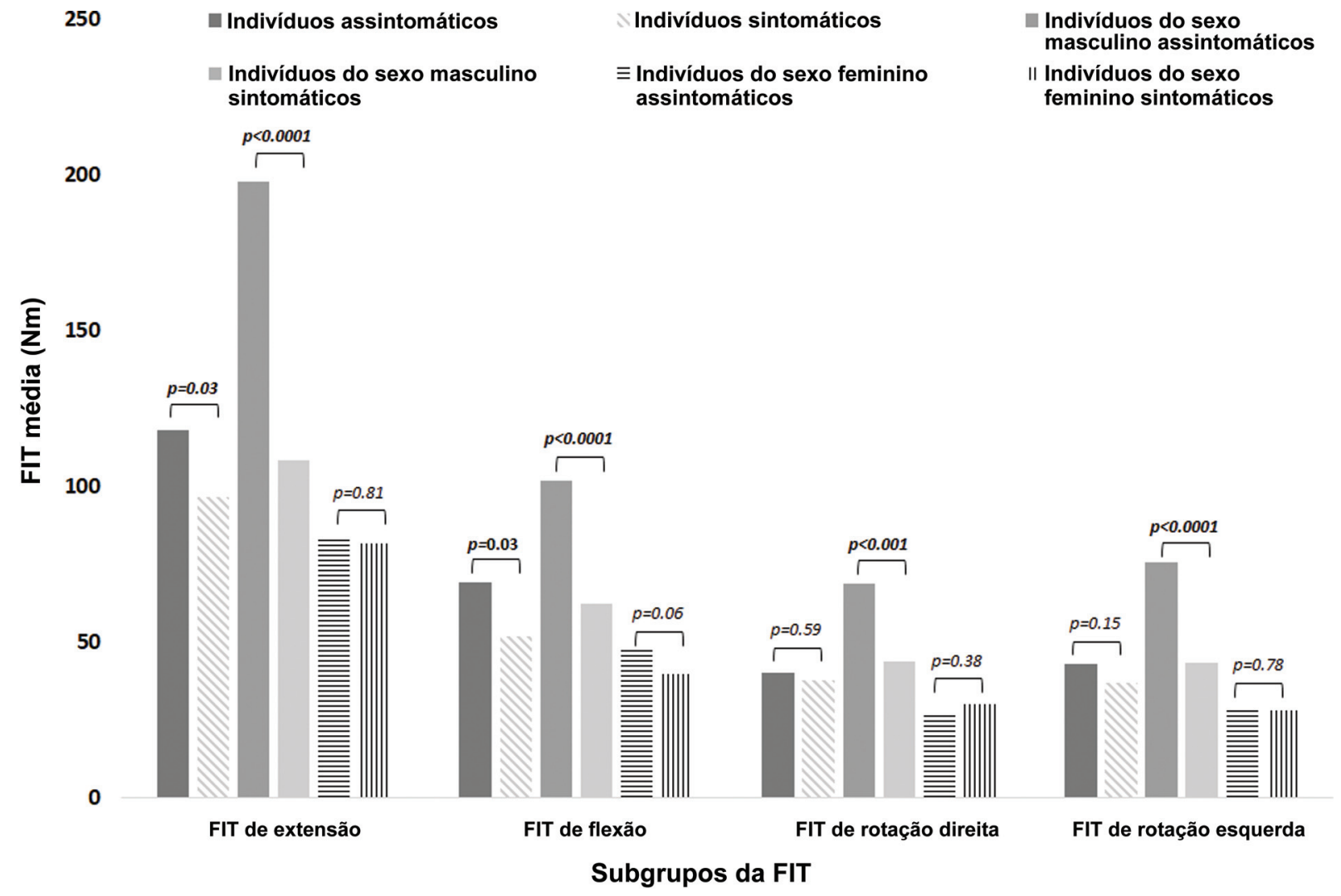

Fig. 2 Comparação da força isométrica do tronco (FIT) média entre indivíduos do sexo masculino e feminino assintomáticos e sintomáticos. $p$ $<0,05$ é considerado estatisticamente significativo (negrito). 
Variações baseadas em gênero em jovens adultos com dor lombar Jain et al. 399
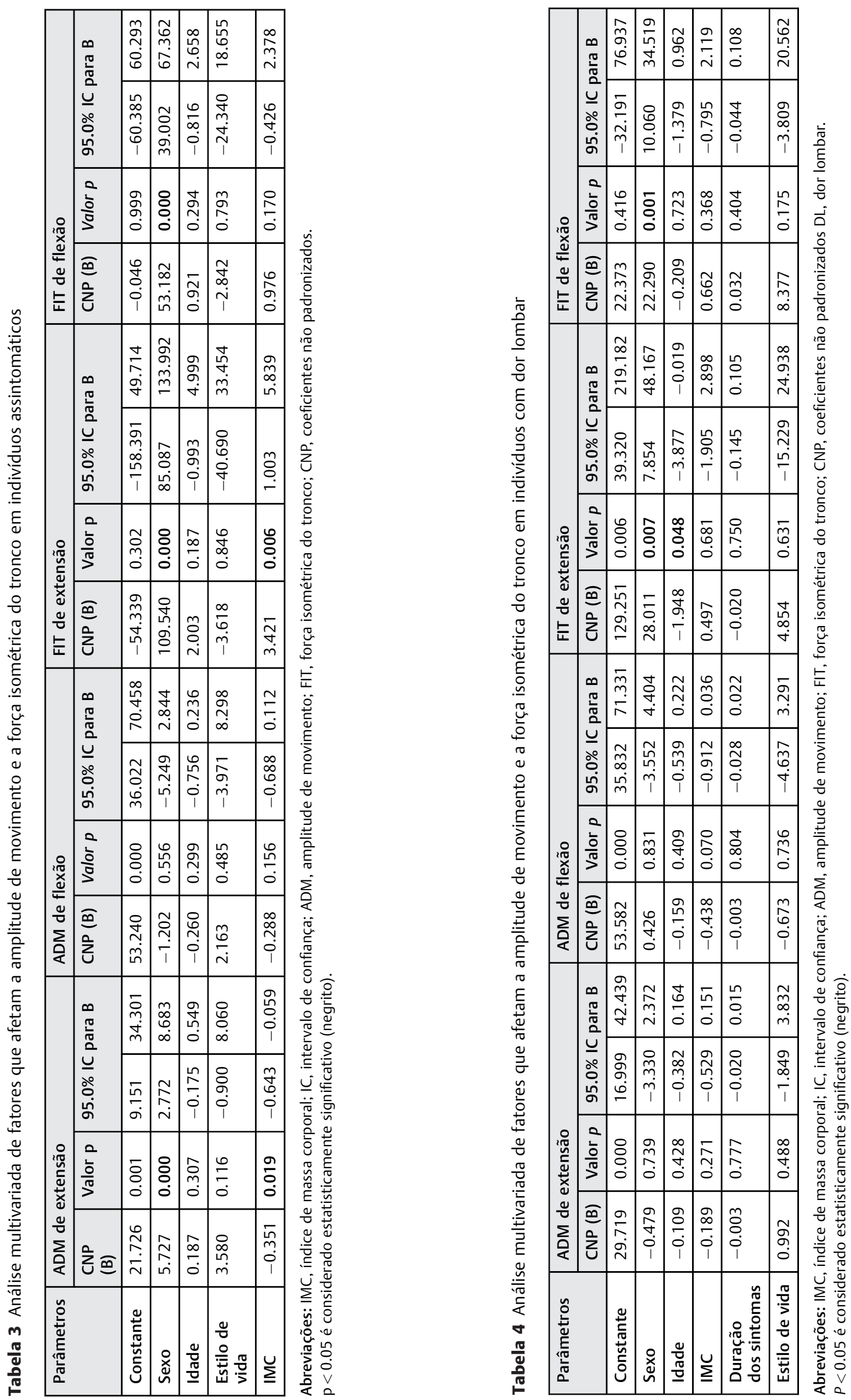
tronco ou dos grupos de músculos flexores pode ser menos problemática do que um desequilíbrio entre os extensores e os flexores do tronco. O desequilíbrio da força do tronco é visto na DL, e foi relatado que aumenta o risco de lesão e dor durante as atividades funcionais em indivíduos com DL. ${ }^{14,15}$ Foi relatado um aumento significativo na razão de pico de torque de flexão-extensão em indivíduos sintomáticos em comparação com indivíduos assintomáticos no grupo masculino e feminino. ${ }^{15}$ Isso destaca a necessidade de corrigir o desequilíbrio da força de extensão-flexão pelo músculo flexor/abdominal do tronco (por exemplo, transverso abdominal) fortalecendo os músculos extensores vis-à-vis (por exemplo, multífidos, eretor da espinha) em indivíduos do sexo feminino com DL. Além disso, a falta de diferença na FIT entre indivíduos do sexo feminino assintomáticos e sintomáticos, em contraste com indivíduos do sexo masculino, indica que grupos musculares individuais fracos podem não ser uma causa ou patologia subjacente de DL em indivíduos do sexo feminino, e outros fatores como IMC e fatores genéticos podem desempenhar um papel neles. ${ }^{16,17}$

A análise multivariada mostrou que sexo feminino e maior IMC foram significativamente associados com menor ADM de extensão do tronco em indivíduos assintomáticos. Embora tenha sido relatado que o sexo feminino tem maior ADM na maioria das articulações, incluindo ADM de flexão do tronco, do que jovens indivíduos do sexo masculino assintomáticos, ${ }^{18}$ nossos achados indicam que isso pode não ser verdade para ADM de extensão. Para a FIT em indivíduos assintomáticos, o sexo feminino foi significativamente associado à FIT de menor extensão e flexão. Um estudo anterior confirmou esses achados, e relatou uma correlação significativa entre o sexo feminino e a FIT de extensão e flexão menor em jovens adultos. ${ }^{19}$ Pajoutana et al. ${ }^{16}$ não relataram correlação significativa entre FIT de extensão e aumento do IMC ou massa gorda do tronco em adultos jovens assintomáticos, o que foi contrário aos achados do nosso estudo. No entanto, indivíduos obesos podem ter torque extensor e flexor do tronco significativamente maior, o que pode ser devido à massa corporal adicional agindo como um estímulo de carga e treinamento nos extensores de tronco antigravitacionais. ${ }^{20,21} \mathrm{O}$ sexo feminino e o aumento da idade como fatores de risco para fraqueza dos músculos extensores e flexores na DL não foram relatados anteriormente em adultos jovens. No entanto, uma associação significativa entre o sexo feminino e o aumento da idade e fraqueza muscular do tronco foi relatada em adultos mais velhos. $^{22}$

O presente estudo tem algumas limitações. Primeiro, indivíduos assintomáticos foram recrutados usando um método de amostragem de conveniência que pode ter causado viés de seleção e, portanto, nossos achados devem ser replicados usando controles da população em geral. Em segundo lugar, o esforço máximo aplicado por indivíduos sintomáticos durante o teste de ADM do tronco e da FIT pode ter sido afetado pelo comportamento de evitação do medo do paciente e tolerância à dor, em vez de sua função muscular. No entanto, levando isso em consideração, garantimos que todos os pacientes sintomáticos fossem testados após a redução da dor para NPRS $<3$ e a melhor de 3 leituras de ADM e da FIT fossem registradas. Finalmente, apesar da determinação da correlação de fatores como gênero e IMC com variações de ADM e FIT, a razão estrutural ou patológica subjacente para ADM e FIT reduzidas em pacientes com DL não pôde ser determinada a partir dos dados coletados no estudo atual. No entanto, estudos anteriores relataram vários fatores, como diferenças no tamanho do músculo do tronco e padrões de recrutamento, ${ }^{23}$ força muscular, resistência e controle de força, ${ }^{10}$ e cinemática lombo-pélvica, ${ }^{14}$ como prováveis causas da diferença na ADM e FIT entre sujeitos com ou sem DL.

\section{Conclusão}

Indivíduos do sexo masculino com DL apresentaram força isométrica do extensor, flexor e rotador significativamente mais fraca em comparação com indivíduos do sexo masculino assintomáticos. Embora não tenha havido diferença significativa na FIT em indivíduos do sexo feminino sintomáticos versus assintomáticos, a DL causou um desequilíbrio significativo na ADM de extensão-flexão e FIT, indicando que os flexores eram mais fracos do que os grupos de músculos extensores no sexo feminino. Tanto em indivíduos sintomáticos quanto assintomáticos, o sexo feminino foi significativamente associado com fraqueza de FIT em extensores e flexores. Essas variações baseadas em gênero na ADM do tronco e na FIT, especialmente o desequilíbrio da FIT do extensor-flexor em indivíduos do sexo feminino, devem ser consideradas ao projetar protocolos de tratamento de reabilitação para DL.

\section{Suporte Financeiro}

Não houve suporte financeiro de fontes públicas, comerciais, ou sem fins lucrativos.

\section{Conflitos de interesses}

Os autores declaram não haver conflito de interesses.

\section{Referências}

1 Cho KH, Beom JW, Lee TS, Lim JH, Lee TH, Yuk JH. Trunk muscles strength as a risk factor for nonspecific low back pain: a pilot study. Ann Rehabil Med 2014;38(02):234-240

2 Kato S, Murakami H, Demura S, et al. Abdominal trunk muscle weakness and its association with chronic low back pain and risk of falling in older women. BMC Musculoskelet Disord 2019;20 (01):273

3 Marcus NJ, Schmidt FA. Soft Tissue: A Possible Source of Pain Pre and Post Minimally Invasive Spine Surgery. Global Spine J 2020; 10(2, Suppl)137S-142S

4 Iwai K, Nakazato K, Irie K, Fujimoto H, Nakajima H. Trunk muscle strength and disability level of low back pain in collegiate wrestlers. Med Sci Sports Exerc 2004;36(08):1296-1300

5 Owen PJ, Miller CT, Mundell NL, et al. Which specific modes of exercise training are most effective for treating low back pain? Network meta-analysis. Br J Sports Med 2020;54(21):1279-1287

6 Bible JE, Biswas D, Miller CP, Whang PG, Grauer JN. Normal functional range of motion of the lumbar spine during 15 activities of daily living. J Spinal Disord Tech 2010;23(02):106-112

7 Sadler SG, Spink MJ, Ho A, De Jonge XJ, Chuter VH. Restriction in lateral bending range of motion, lumbar lordosis, and hamstring flexibility predicts the development of low back pain: a 
systematic review of prospective cohort studies. BMC Musculoskelet Disord 2017;18(01):179

8 Kienbacher T, Fehrmann E, Habenicht R, et al. Diagnostic value of trunk flexion-extension testing in old chronic low back pain patients. Eur Spine J 2017;26(02):510-517

9 Verbrugghe J, Agten A, Eijnde BO, et al. Reliability and agreement of isometric functional trunk and isolated lumbar strength assessment in healthy persons and persons with chronic nonspecific low back pain. Phys Ther Sport 2019;38(07):1-7

10 Pranata A, Perraton L, El-Ansary D, et al. Lumbar extensor muscle force control is associated with disability in people with chronic low back pain. Clin Biomech (Bristol, Avon) 2017;46(07):46-51

11 Will JS, Bury DC, Miller JA. Mechanical Low Back Pain. Am Fam Physician 2018;98(07):421-428

12 Childs JD, Piva SR, Fritz JM. Responsiveness of the numeric pain rating scale in patients with low back pain. Spine (Phila Pa 1976) 2005;30(11):1331-1334

13 Kienbacher T, Paul B, Habenicht R, et al. Reliability of isometric trunk moment measurements in healthy persons over 50 years of age. J Rehabil Med 2014;46(03):241-249

14 Ruas CV, Vieira A. Do Muscle Strength Imbalances and Low Flexibility Levels Lead to Low Back Pain? A Brief Review. J Funct Morphol Kinesiol 2017;2(03):29-38

15 Shirado O, Ito T, Kaneda K, Strax TE. Concentric and eccentric strength of trunk muscles: influence of test postures on strength and characteristics of patients with chronic low-back pain. Arch Phys Med Rehabil 1995;76(07):604-611
16 Pajoutana M, Mehta RK, Cavuotoa LA. Obesity effect on isometric strength of the trunk extensors. Proc Hum Factors Ergon Soc Annu Meet 2016;60:943-947

17 Battié MC, Levalahti E, Videman T, Burton K, Kaprio J. Heritability of lumbar flexibility and the role of disc degeneration and body weight. J Appl Physiol (1985) 2008;104(02):379-385

18 Moromizato K, Kimura R, Fukase H, Yamaguchi K, Ishida H. Wholebody patterns of the range of joint motion in young adults: masculine type and feminine type. J Physiol Anthropol 2016;35(01):23

19 Skrzek A, Ignasiak Z, Kozieł S, Sławińska T, Rożek K. Differences in muscle strength depend on age, gender and muscle functions. Isokinet Exerc Sci 2012;20(03):229-235

20 Hulens M, Vansant G, Lysens R, Claessens AL, Muls E, Brumagne S. Study of differences in peripheral muscle strength of lean versus obese women: an allometric approach. Int J Obes Relat Metab Disord 2001;25(05):676-681

21 Tomlinson DJ, Erskine RM, Morse CI, Winwood K, OnambéléPearson G. The impact of obesity on skeletal muscle strength and structure through adolescence to old age. Biogerontology 2016; 17(03):467-483

22 Sasaki E, Sasaki S, Chiba D, et al. Age-related reduction of trunk muscle torque and prevalence of trunk sarcopenia in communitydwelling elderly: Validity of a portable trunk muscle torque measurement instrument and its application to a large sample cohort study. PLoS One 2018;13(02):e0192687

23 Goubert D, Oosterwijck JV, Meeus M, Danneels L. Structural Changes of Lumbar Muscles in Non-specific Low Back Pain: A Systematic Review. Pain Physician 2016;19(07):E985-E1000 